\title{
Influência da mídia em universitárias brasileiras de diferentes regiões
}

\author{
Media influence in female university students in all Brazilian regions \\ Marle dos Santos Alvarenga ${ }^{1,2}$, Karin Louise Lenz Dunker, Sonia Tucunduva Philippi', \\ Fernanda Baeza Scagliusi ${ }^{4}$
}

\section{RESUMO}

A mídia tem impacto na satisfação com a imagem corporal e risco para o desenvolvimento de transtornos alimentares. Objetivo: Avaliar a influência da mídia em universitárias e possíveis associações com idade, estado nutricional, renda e escolaridade do chefe da família. Métodos: 2.489 estudantes do sexo feminino das cinco regiões do Brasil responderam à Sociocultural Attitudes Towards Appearance Scale (SATAQ-3). O escore na SATAQ foi comparado entre as regiões por meio de uma análise de variância. Uma análise de covariância foi utilizada para verificar a influência das variáveis estudadas no escore da SATAQ. Uma regressão logística foi realizada para verificar a interferência conjunta das variáveis em relação à influência da mídia. Resultados: Não foram encontradas diferenças regionais na SATAQ total ( $p=$ $0,164)$ e subescalas Internalização atlética $(p=0,293)$ e Pressão $(p=0,150)$; houve diferença para as subescalas Internalização geral $(p=0,010)$ e Informação $(p=0,002)$. Idade, estado nutricional e renda influenciaram o resultado. Conclusões: $O$ escore total na SATAQ foi similar entre as regiões, mas o Sul e o Nordeste apresentaram maiores pontuações para subescalas Internalização geral e Informação respectivamente. Estudantes com menos de 25 anos, com excesso de peso e maior renda foram em média mais influenciadas pela mídia.

\section{ABSTRACT \\ Media has been postulated to be a causal risk factor for body dissatisfaction and increases in eating disorder symptoms. Objective: To evaluate the media influence on Brazilian undergraduate stu- dents and possible associations with age, nutritional status, income and education of family head. Methods: 2.489 female students from five regions of Brazil answered the "Sociocultural Attitudes Towards Appearance Scale" (SATAQ-3) and its scores among regions were compared by means of a variance analysis. A covariance analysis was performed to evaluate the possible effect of stud- ied variables in the scores. A logistic regression was done to evaluate association of variables with the media influence. Results: There was no regional differences in SATAQ total score ( $p=0.164)$, and subscales Internalization-athlete $(p=0.293)$ and Pressure $(p=0.150)$; there was difference for subscales Internalization-general $(p=0.010)$ e Information $(p=0.002)$. Age, nutritional status and}

1 Universidade de São Paulo (USP), Faculdade de Saúde Pública (FSP), Departamento de Nutrição. 2 USP, Faculdade de Medicina, Instituto de Psiquiatria, Programa de Transtornos Alimentares (Ambulim). 


\section{Keywords}

Media influence, appearance, dissatisfaction, university students. income influenced the result. Conclusions: The SATAQ total score were similar among regions, but it was noticed that the South and Northeast regions of Brazil presented the highest scores at Internalization-general and Information subscales. Students under 25 years old, overweight and those with higher income were more influenced by media.

\section{INTRODUÇÃO}

O conceito "a mídia é a mensagem" engloba o fato de que as novas tecnologias exercem um efeito nas cognições, que por sua vez afetam a percepção de hábitos e interações sociais ${ }^{1}$. A influência ou efeito da mídia seria o modo pelo qual os meios de comunicação em massa afetam o comportamento e o pensamento da sua audiência'. A mídia de massa tem um papel crucial na formação e reflexão da opinião pública, reproduzindo a autoimagem da sociedade'. Acredita-se que ela pode influenciar valores, normas e padrões estéticos incorporados pela sociedade moderna². A mídia de massa é um transmissor e reforçador dos ideais sociais corporais ${ }^{3}$.

Alguns trabalhos discutem as evidências do papel da mídia no desenvolvimento das desordens alimentares, e a imagem e insatisfação corporal seriam os elos mais importantes dessa relação 24,5 ; a leitura de revistas, a exposição a figuras de corpos magros (em fotos, filmes) e o assistir à televisão têm impacto na insatisfação corporal, e esse impacto é maior para adolescentes e mulheres jovens - em países desenvolvidos e em desenvolvimento $2,4,5$.

Transtornos alimentares (TA) são desordens psiquiátricas que afetam de $0,5 \%$ a 3,0\% da população, sendo mais prevalentes em mulheres ${ }^{6}$. Os quadros atípicos ou subclínicos (chamados TA não especificados) são mais prevalentes do que os típicos - anorexia e bulimia nervosa -, e comportamentos de risco ou comer desordenado são ainda mais frequentes em mulheres jovens ${ }^{6}$. Entre estudantes universitárias, vários estudos apontam comportamentos de TA variando entre 5,5\% e 40\% dessa população 6 .

A etiologia dos TA é multifatorial; aspectos biológicos, psicológicos, familiares e socioculturais se somam. As mudanças nas normas socioculturais nos últimos 40 anos sugerem que os fatores socioculturais têm um papel no aumento da prevalência de TA observado nas últimas décadas².

A prevenção da obesidade tem sido sugerida como importante estratégia de saúde pública e também a prevenção dos TA é colocada como emergência em saúde ${ }^{7,8}$ : pelo aumento da demanda e opções limitadas de tratamento (além do custo), cronicidade dos casos e recaídas frequentes. Considerando a etiologia multifatorial dos TA, os fatores socioculturais são aqueles nos quais se pode interferir, portanto avaliar a influência da mídia é importante para o planejamento de estratégias de prevenção e compreensão dos fatores relacionados aos comportamentos inadequados para com a alimentação e a insatisfação corporal ${ }^{2,7}$.
Nesse contexto, o objetivo deste estudo foi avaliar a influência da mídia em estudantes universitárias do sexo feminino nas cinco diferentes regiões do Brasil e avaliar também possíveis associações com a idade, estado nutricional, renda individual e escolaridade do chefe da família.

\section{MÉTODOS}

Participaram da pesquisa estudantes de instituições de ensino superior convidadas para uma parceria por meio de convites via e-mail. Foram enviados convites a 130 instituições, dentre as quais $37(28,5 \%)$ aceitaram participar.

A pesquisa global teve como objetivo avaliar atitudes alimentares, comportamento de risco para TA, imagem corporal e influência da mídia. O tamanho amostral foi definido a partir da frequência hipotética de atitudes alimentares desordenadas nessa população - definida como um mínimo de 117 sujeitos por região9. Os critérios de inclusão foram: cursar o primeiro ou o segundo ano de enfermagem, psicologia, fisioterapia, farmácia, fonoaudiologia ou biomedicina (os cursos da área da saúde mais frequentes nas instituições foram escolhidos para padronização da amostra); ser do gênero feminino; ter idade entre 18 e 50 anos; assinar o termo de consentimento. Os critérios de exclusão foram: ser nutricionista ou cursar nutrição (porque alguns trabalhos mostram atitudes alimentares piores nesse público); estar grávida ou informar alguma condição que pudesse ter impacto em atitudes alimentares - como ter um TA9 .

A amostra total incluiu 2.489 estudantes, sendo 9,6\% da região Norte, $15,4 \%$ do Nordeste, 8,0\% do Centro-Oeste, $35,6 \%$ do Sudeste e $31,4 \%$ do Sul. Dentre os cursos de graduação, 58,7\% cursavam enfermagem; 15,1\%, psicologia; 12,3\%, farmácia, 9,4\%, fisioterapia; 1,8\%, biomedicina e 1,3\%, fonoaudiologia - 1,4\% não informou o curso.

\section{INSTRUMENTO}

A influência da mídia foi avaliada por meio da Sociocultural Attitudes Towards Appearance Scale (SATAQ), a terceira versão deste instrumento - utilizada neste estudo (SATAQ-3) - foi desenvolvida com uma amostra de universitárias após algumas revisões do instrumento original ${ }^{10}$. Ela possui um total de 30 questões numa escala Likert (Alpha de Cronbach = 0,94) e possui quatro subescalas: subescala 1 - Internalização 
geral: avalia a aceitação das mensagens da mídia em relação a ideais estéticos não realistas; subescala 2 - Internalização atlética: avalia a adesão e a aceitação de um corpo ideal atlético; subescala 3 - Pressão: avalia a pressão de várias mídias para "batalhar" pelos ideais de beleza; e subescala 4 - Informação: avalia a extensão na qual várias mídias são consideradas uma importante fonte de informação sobre a aparência.

As opções de resposta são: concordo fortemente, concordo, sou neutro, discordo e discordo fortemente $(5,4,3$, 2 e 1 pontos, respectivamente). A versão em português foi elaborada com autorização do autor original e realizada por meio de um processo de tradução e retrotradução ${ }^{11}$. O instrumento demonstrou boa consistência interna na avaliação desse grupo de universitárias (Alpha de Cronbach =0,91).

As instituições receberam instruções sobre a administração dos instrumentos, que foram autopreenchidos em sala de aula, respeitando-se os critérios de inclusão e exclusão. As participantes também informaram idade, peso e altura (autorreferidos), renda individual e educação do chefe da família. A faixa etária foi classificada em três categorias: até 19 anos, de 20-24 anos, maior de 25 anos. O estado nutricional foi classificado de acordo com as categorias do índice de massa corporal (IMC) da Organização Mundial da Saúde ${ }^{12}$ e analisado como: baixo peso (IMC $\left.<18,49 \mathrm{~kg} / \mathrm{m}^{2}\right)$, peso normal ou eutrofia (IMC 18,50-24,99 $\left.\mathrm{kg} / \mathrm{m}^{2}\right)$ e acima do peso $\left(\mathrm{IMC}>25,0 \mathrm{~kg} / \mathrm{m}^{2}\right)$. A renda individual foi analisada em faixas de salários-mínimos (SM) nas categorias: $<1,9$ SM, de 2 a 4,9 SM, de 5 a 9,9 SM e > 10 SM; e a escolaridade do chefe da família, nas categorias: analfabeto ou até a quarta série primária, fundamental, secundário e superior.

\section{Análise dos dados}

As análises estatísticas foram conduzidas pelo software SPSS 12.0 (Statistical Package for Social Science Inc., Chicago, Illinois USA). O nível de significância adotado foi 0,05. A normalidade das distribuições das variáveis foi testada por meio do teste de Kolmogorov-Smirnov.

Os resultados na SATAQ nas regiões foram comparados entre si por meio da análise de variância (ANOVA). Foi verificado se as variâncias eram homogêneas e, quando esta não foi verificada, foi feito o ajuste por meio do teste de BrownForsythe. Uma análise de covariância foi utilizada para verificar se a idade, o IMC, a renda e a escolaridade poderiam influenciar nos resultados. Caso a variável de interesse não apresentasse significância no modelo, seguiu-se a análise com a ANOVA, excluindo-se essas covariáveis. Para a identificação de quais grupos apresentam diferenças entre si, foram feitas comparações múltiplas (dois a dois) utilizando o teste de Tukey.

Os escores totais da SATAQ foram classificados em duas categorias (maiores e menores valores) por meio da mediana dos resultados, e uma comparação entre elas foi realizada por meio do teste do qui-quadrado para identificar se aquelas menos ou mais influenciadas pela mídia se diferenciavam em relação a renda, escolaridade, idade e IMC.
Uma análise de regressão logística foi realizada para verificar a interferência conjunta das variáveis em relação à influência da mídia; foram inseridas no modelo inicial aquelas que apresentaram nível de significância abaixo de 0,10 nas comparações univariadas das categorias de pontuação maior e menor na SATAQ, utilizando o método stepwise forward - que não inclui no modelo final aquelas que não têm significância estatística.

\section{Aspectos éticos}

As universitárias preencheram o termo de consentimento livre e esclarecido. A pesquisa foi aprovada pelo Comitê de Ética da Faculdade de Saúde Pública da Universidade de São Paulo.

\section{RESULTADOS}

Foram enviados 2.925 instrumentos às instituições parceiras e recebidos 2.547 preenchidos. Alguns foram excluídos $(2,3 \%)$, porque estavam incompletos ou respondidos por estudantes que não preenchiam os critérios de inclusão. A amostra total incluiu 2.489 estudantes, destes 2.414 responderam adequadamente à SATAQ. A resposta à SATAQ (total e subescalas) de acordo com a faixa etária, estado nutricional, renda, escolaridade e as regiões do país pode ser visualizada na tabela 1.

Houve diferença entre as regiões para a subescala 1 (SUB1) e a subescala 4 (SUB4). Para a SUB1, o IMC, renda e idade mostraram efeito e o modelo ajustado mostrou que a diferença se manteve; as comparações múltiplas evidenciaram que em média o Sul teve maior pontuação para Internalização geral. Nenhuma das covariáveis foi significante no modelo da SUB4, e as comparações múltiplas pós-ANOVA evidenciaram que houve diferença, com o Nordeste apresentando maior pontuação para Informação.

Para escore total na SATAQ, o IMC, a renda e a idade tiveram efeito no resultado, mas, quando ajustado para essas variáveis, a diferença não se manteve. O IMC e a renda tiveram efeito sobre o resultado das subescalas 2 (SUB2) e 3 (SUB3), mas o modelo ajustado não manteve a diferença.

Em relação à faixa etária, a análise de variância apontou diferença para o escore total e para SUB1. As comparações múltiplas mostraram que as estudantes mais velhas apresentam, em média, menor pontuação total e menor Internalização geral. Quanto ao estado nutricional, observou-se que quanto maior o IMC, maior a pontuação total na SATAQ e na subescala Pressão e que estudantes com baixo peso têm menor Internalização geral e Internalização atlética. Em relação à renda, a análise de variância apontou diferença para o escore total, SUB1 e SUB2, e estudantes com menor renda apresentaram menor pontuação total, menor Internalização geral e atlética. Por fim, quanto à escolaridade do chefe da família, não houve diferença entre as categorias. 
Tabela 1. Pontuação da SATAQ (total e quatro subescalas) da amostra de universitárias brasileiras, segundo faixa etária, estado nutricional, renda, escolaridade do chefe da família e regiões do país

\begin{tabular}{|c|c|c|c|c|c|}
\hline & Total & SUB1 & SUB2 & SUB3 & SUB4 \\
\hline \multicolumn{6}{|l|}{ Faixa etária } \\
\hline$<19$ anos & $84,9(18,8)$ & $24,5(7,8)$ & $13,8(3,8)$ & $18,5(6,5)$ & $28,1(6,3)$ \\
\hline 20-24 anos & $86,2(19,3)^{\mathrm{a}}$ & $24,9(7,6)$ & $13,7(3,6)$ & $19,1(6,5)$ & $28,5(6,6)$ \\
\hline$>25$ anos & $83,8(18,2)$ & $23,1(7,1)^{b}$ & $13,4(3,6)$ & $18,9(6,3)$ & $28,4(6,2)$ \\
\hline$p$ & 0,037 & $<0,001$ & 0,091 & 0,145 & 0,461 \\
\hline \multicolumn{6}{|l|}{ Estado nutricional } \\
\hline Baixo peso & $79,2(18,0)$ & $22,6(7,7)^{d}$ & $12,8(3,7)^{\mathrm{e}}$ & $15,8(5,5)$ & $28,0(6,7)$ \\
\hline Eutrofia & $85,3(18,7)$ & $24,4(7,6)$ & $13,7(3,6)$ & $18,9(6,4)$ & $28,4(6,4)$ \\
\hline Excesso de peso & $89,1(18,7)^{c}$ & $25,2(7,5)$ & $13,9(3,7)$ & $21,1(6,6)^{f}$ & $28,9(6,2)$ \\
\hline$p$ & $<0,001$ & $<0,001$ & 0,001 & $<0,001$ & 0,209 \\
\hline Renda & \multicolumn{5}{|c|}{$\mathrm{SM}=$ salários-mínimos } \\
\hline$<1,9 \mathrm{SM}$ & $84,2(19,0)^{9}$ & $23,8(7,5)^{\mathrm{h}}$ & $13,5(3,6)^{i}$ & $18,7(6,5)$ & $28,2(6,3)$ \\
\hline 2 a 4,9 SM & $86,6(18,3)$ & $24,8(7,5)$ & $14,0(3,7)$ & $19,3(6,3)$ & $28,5(6,7)$ \\
\hline 5,0 a $9,9 \mathrm{SM}$ & $85,8(20,4)$ & $24,6(8,2)$ & $13,6(3,7)$ & $18,9(7,2)$ & $28,6(6,3)$ \\
\hline$>10 \mathrm{SM}$ & $95,5(22,1)$ & $26,9(9,4)$ & $15,1(4,1)$ & $20,8(8,1)$ & $29,7(7,3)$ \\
\hline$p$ & 0,004 & 0,005 & 0,002 & 0,104 & 0,385 \\
\hline \multicolumn{6}{|l|}{ Escolaridade } \\
\hline $\begin{array}{l}\text { Analfabeto ou primário } \\
\text { incompleto }\end{array}$ & $86,8(19,7)$ & $24,3(7,7)$ & $13,9(3,9)$ & $19,8(6,5)$ & $28,8(6,3)$ \\
\hline Fundamental completo & $84,5(18,6)$ & $23,8(7,4)$ & $13,4(3,7)$ & $19,0(6,4)$ & $28,3(6,2)$ \\
\hline $\begin{array}{l}\text { Secundário completo ou } \\
\text { incompleto }\end{array}$ & $84,4(18,6)$ & $24,0(7,5)$ & $13,6(3,6)$ & $18,5(6,5)$ & $28,2(6,3)$ \\
\hline $\begin{array}{l}\text { Superior completo ou } \\
\text { incompleto }\end{array}$ & $85,7(19,0)$ & $24,6(7,7)$ & $13,7(3,7)$ & $19,0(6,5)$ & $28,5(6,5)$ \\
\hline$p$ & 0,299 & 0,272 & 0,484 & 0,213 & 0,666 \\
\hline \multicolumn{6}{|l|}{ Regiões } \\
\hline Norte $(n=225)$ & $83,3(17,3)$ & $23,4(6,5)$ & $13,4(3,5)$ & $18,4(6,2)$ & $28,2(5,8)$ \\
\hline Nordeste $(n=368)$ & $85,0(17,4)$ & $23,6(7,0)$ & $13,4(3,4)$ & $18,6(6,1)$ & $29,5(6,2)^{\mathrm{k}}$ \\
\hline Centro-0este $(n=191)$ & $86,3(18,5)$ & $24,7(7,4)$ & $14,0(3,9)$ & $18,9(6,3)$ & $28,8(6,3)$ \\
\hline Sudeste $(n=863)$ & $84,7(19,2)$ & $24,1(7,8)$ & $13,6(3,7)$ & $18,7(6,6)$ & $28,2(6,4)$ \\
\hline $\operatorname{Sul}(n=767)$ & $86,0(19,5)$ & $24,9(7,8)^{j}$ & $13,8(3,7)$ & $19,3(6,6)$ & $28,0(6,6)$ \\
\hline Total nacional & $85,2(18,8)$ & $24,3(7,5)$ & $13,64(3,7)$ & $18,9(6,5)$ & $28,4(6,4)$ \\
\hline$p$ & 0,164 & 0,010 & 0,293 & 0,150 & 0,002 \\
\hline
\end{tabular}

Nota: Dados apresentados como média (desvio-padrão).

a: diferente de > 25 anos; b: diferente de < 19 e $20-24$ anos; c: diferente de baixo peso e eutrofia; d: diferente de eutrofia e excesso de peso; e: diferente de eutrofia e excesso de peso; $f$ : diferente de baixo peso e eutrofia; $\mathrm{g}$ : diferente de 2 a 4,9 SM e > 10 SM; $h$ : diferente de 2 a 4,9 SM e > 10 SM; i: diferente de 2 a 4,9 SM e > 10 SM; j: diferente do Norte e Nordeste; $k$ : diferente do Sul e Sudeste.

A comparação entre as categorias de pontuação mais alta e mais baixa na SATAQ (mediana = escore 85) encontrou 49,6\% ( $n=1.198$ ) das estudantes com valores menores e $50,4 \%$ ( $n=1.216$ ) com valores maiores. Observou-se que no grupo com maior influência da mídia há maior percentual de estudantes com excesso de peso e no grupo com menor influência da mídia há um maior percentual de estudantes com renda menor do que 1,9 SM e com mais de 25 anos.
A regressão logística evidenciou que universitárias com mais de 25 anos têm 23\% [(1-0,77)*100] menos chance de serem influenciadas pela mídia do que aquelas com até 19 anos; que estudantes com peso normal têm 1,77 vez mais chance de serem influenciadas pela mídia do que aquelas com baixo peso; e que estudantes com excesso de peso têm 1,74 vez mais chance de serem influenciadas pela mídia do aquelas com baixo peso. 


\section{DISCUSSÃO}

Este trabalho avaliou a influência da mídia em universitárias brasileiras e não encontrou diferenças regionais quanto ao escore total no questionário utilizado. No entanto, estudantes da região Sul mostraram maior aderência e aceitação de ideais estéticos não realistas do que as do Norte e Nordeste, e as do Nordeste consideram a mídia uma fonte de informação importante sobre a aparência, mais do que as do Sul e Sudeste. Encontrou-se ainda que as mais novas, com excesso de peso e com maior renda são em média mais influenciadas pela mídia.

Os dados gerais sobre essa população evidenciaram que, em todas as regiões, as participantes eram jovens (69,8\% entre 18-24 anos), com peso normal $(74,6 \%)$ e com renda mensal menor do que 2 SM (57,1\%); e esse perfil é semelhante ao relatado para mulheres dessa faixa etária no Brasil ${ }^{9}$. Esse grupo de estudantes é, portanto, uma boa amostra de muIheres jovens brasileiras.

O escore total na SATAQ evidenciou que a influência da mídia para essas mulheres é igual mesmo elas vivendo em realidades culturais diferentes. Os hábitos alimentares, as experiências culturais e o estilo de vida são sabidamente diferentes nas cinco regiões brasileiras, mesmo assim se encontrou resposta similar quanto à influência da mídia como um todo, à adesão e aceitação de um corpo ideal atlético e à pressão de várias mídias pelos ideais de beleza. Esse resultado de similaridades faz sentido quando se observa que a população brasileira é exposta aos mesmos programas de televisão, revistas, filmes, modismos e notícias; isso é ainda especialmente verdadeiro para universitárias, que - em virtude da idade e condição sociocultural semelhante - têm acesso ao mesmo tipo de informação e são sujeitas aos mesmos padrões para corpo e aparência.

Mesmo sendo a influência da mídia similar, as diferenças em relação a Internalização geral e informação evidenciam que algumas diferenças regionais existem e devem ser consideradas em relação à imagem corporal. A internalização é conceituada como a incorporação de valores específicos, de forma a se tornarem princípios e guias, ou a extensão em que os indivíduos cognitivamente "compram" as normas da sociedade em relação à aparência, a ponto de modificarem seu comportamento na tentativa de se aproximarem desses padrões ${ }^{10,13}$.

Não há dados específicos sobre mídia nas diferentes regiões do país que permitam um entendimento em profundidade dessas diferenças; dessa forma, não é fácil entender exatamente por que estudantes do Sul apresentaram maior aderência e aceitação de ideais estéticos não realistas do que as do Norte e Nordeste (e por que isso não foi válido também para o Sudeste - semelhante em termos de "desenvolvimento"). Também não é simples entender por que as do Nordeste consideram a mídia uma fonte de informação sobre a aparência mais importante do que as do Sul e Sudeste (e por que isso também não foi verdadeiro para o Norte - região mais próxima socioculturalmente).

Não há uma tradição de comparação de dados nas regiões do Brasil em relação a parâmetros que vão além de renda, escolaridade e estado nutricional. Assim, crenças, atitudes e modos de pensar e viver não são sistematicamente avaliados. De qualquer forma, existe uma percepção generalizada de que os estados do Centro-Sul são mais "desenvolvidos" e se imaginaria, portanto, que apresentassem piores parâmetros em relação à imagem corporal, comportamento de risco para TA e métodos inadequados para controle e perda de peso. Essa percepção pode ser equivocada e prejudicar estratégias educacionais e terapêuticas.

Trabalhos sobre imagem corporal são escassos no país. Alvarenga et al. ${ }^{14}$, comparando regiões, encontraram que os ideais mais magros de aparência estavam "surpreendentemente" no Norte. Em relação aos trabalhos sobre comportamento de risco para TA, estes são localizados e com públicos muito diversificados; o único trabalho comparativo entre regiões do país não encontrou diferença entre universitárias nas cinco regiões ${ }^{15}$. Na avaliação de atitudes alimentares nessas mesmas universitárias, as atitudes mais desordenadas foram encontradas no Norte e Nordeste ${ }^{15}$; é preciso, portanto, que se olhe para as regiões do país de forma ampla e não estereotipada em relação a esses aspectos.

A influência da mídia em relação aos demais parâmetros avaliados encontrou resultados consistentes com o conhecimento sobre este aspecto: que as mais novas, com excesso de peso e com maior renda são mais influenciadas. Em relação à idade, a diferença foi em relação ao escore total e Internalização geral, mostrando, portanto, que, em termos de ideais atléticos, pressão e informação, todas as faixas etárias têm a mesma influência. As mais novas parecem aceitar e aderir meIhor aos ideais estéticos não realistas (internalização), e as mais velhas têm menos chance de serem influenciadas pela mídia.

Groez et al..$^{16}$ encontraram em estudo de metanálise que o efeito de manipulações experimentais do ideal de beleza pela mídia também teve efeito maior em participantes com menos de 19 anos; e Madanat et al. ${ }^{17}$ também encontraram que mulheres mais velhas tiveram menores escores na SATAQ.

Em relação ao estado nutricional, a diferença só não foi encontrada para SUB4, mostrando que todas têm a mídia como importante fonte de informação, mas as de baixo peso internalizam menos os ideais atléticos e estéticos irreais; encontrou-se ainda que quanto maior o IMC, maior a pressão sofrida das várias mídias para alcançar os ideais de beleza e maior a influência da mídia como um todo. Esse dado é compreendido quando se observa que a obesidade e o excesso de peso são altamente estigmatizados e associados com atributos como "preguiça" e "falta de vontade"18. Uma observação atenta das revistas femininas e programas de televisão evidencia como o tema da perda de peso e busca do "corpo ideal" é popular e predispõe a uma maior preocupação com o corpo, uso de 
dietas e comportamentos compensatórios - o que ajuda a entender que indivíduos mais distantes do ideal sociocultural sintam-se piores e mais influenciados e pressionados ${ }^{19,20}$.

A comparação entre as categorias de pontuação alta e baixa na SATAQ reforça a maior influência da mídia para as mais velhas e com excesso de peso; esses resultados, no entanto, são pontuais; não há uma relação linear indicando que quanto maior a idade, menor a pontuação, ou quanto maior o IMC, maior a pontuação.

Encontrou-se que estudantes eutróficas têm uma chance maior do que aquelas com excesso de peso de serem influenciadas pela mídia - em relação àquelas com baixo peso. Esse dado relembra que a insatisfação corporal é muito presente mesmo em mulheres de peso normal, uma situação denominada "descontentamento normativo" da sociedade contemporânea, em que todas acreditam que ficariam mais bonitas e atraentes se perdessem algum peso ${ }^{21}$. Nesse contexto, todas as mulheres podem ser influenciadas pela mídia se estão em busca de modelos de corpo e formas de como alcançá-lo - e se essa mídia vende um número infinito de fórmulas para se "conseguir" o corpo desejado.

Em relação à renda, não houve diferença para Informação e Pressão, demonstrando que todos os estratos são pressionados e consideram a mídia importante fonte de informação. No entanto, as com menor renda apresentaram menor influência da mídia em geral e as com maior renda, maior internalização dos ideais atléticos e estéticos irreais. Dunker et al. ${ }^{22}$ também verificaram associação de maior escore na
SATAQ e maior renda. Esses dados confirmariam a ideia geral de que o ideal de beleza se aplica mais incisivamente a indivíduos de estratos econômicos mais privilegiados, no entanto não se encontrou nenhuma diferença em relação à escolaridade do chefe da família - como essa variável também pode ser um indicativo da condição socioeconômica, deve-se interpretar esse achado com cautela, uma vez que a internalização dos padrões socioculturais de beleza é comprovada em diferentes etnias e classes sociais, incluindo adolescentes provenientes de estratos menos favorecidos ${ }^{23}$.

Estudos internacionais avaliando universitárias encontraram que houve um efeito direto da exposição à mídia em sintomas de TA e, entre outros, a internalização do estereótipo do corpo ideal teve um papel de mediação nesse mecanismo ; e que a ansiedade sobre a aparência, bem como a vergonha do próprio corpo, aumentou depois de ver propagandas com imagens idealizadas de beleza ${ }^{24}$. Um estudo de avaliação da influência da mídia em colegiais e jovens adultas na Índia encontrou que a internalização dos ideais da mídia tem papel na insatisfação corporal e busca pela magreza mesmo em um país em desenvolvimento e que, portanto, deve haver fatores de risco potencialmente similares em diferentes culturas ${ }^{25}$.

O número de estudos realizados sobre esse tema utilizando a SATAQ não é muito extenso (Quadro 1). O'Riordan e Zamboanga ${ }^{26}$ avaliaram universitárias americanas e encontraram escores médios menores que os do presente estudo para todas essas subescalas. Madanat et al. ${ }^{17}$ encontraram na

Quadro 1. Resultados na SATAQ - total e subescalas - (apresentados como média e desvio-padrão) e/ou consistência interna (Alpha de Cronbach $\alpha$ ) segundo estudos internacionais e nacionais

\begin{tabular}{|c|c|c|c|c|c|c|}
\hline Autor/Local do estudo & População de estudo (n) & Total & SUB1 & SUB2 & SUB3 & SUB4 \\
\hline Thompson et al. $.^{13}-$ EUA & $\begin{array}{l}175 \text { q universitárias } \\
\text { (17-25 anos) }\end{array}$ & $\alpha=, 94$ & $\alpha=, 96$ & $\alpha=, 95$ & $\alpha=, 92$ & $\alpha=, 96$ \\
\hline $\begin{array}{l}\text { Madanat et al. }{ }^{17} \text { - } \\
\text { Jordânia }\end{array}$ & $\begin{array}{l}100+ \\
\text { (18-60 anos) }\end{array}$ & ND & $\begin{array}{l}21,6 \pm 4,5 \\
\alpha=, 88\end{array}$ & $\begin{array}{l}12,5 \pm 4,0 \\
\alpha=, 82\end{array}$ & $\begin{array}{l}16,8 \pm 4,5 \\
\alpha=, 84\end{array}$ & $\begin{array}{l}24,6 \pm 6,7 \\
\alpha=, 89\end{array}$ \\
\hline Wilksch et al. ${ }^{30}$ - EUA & $\begin{array}{l}100 \text { q } \\
\text { (média de } 14 \text { anos) } \\
\text { (fase de pré-inter- } \\
\text { venção) }\end{array}$ & $78,3 \pm 9,2$ & $24,4 \pm 10,5$ & $12,4 \pm 5,2$ & $18,4 \pm 8,4$ & $23,0 \pm 9,5$ \\
\hline $\begin{array}{l}0^{\prime} \text { Riordan e } \\
\text { Zamboanga }{ }^{26}-\text { EUA }\end{array}$ & $\begin{array}{l}106 \text { }+ \text { universitárias } \\
\text { (18-22 anos) }\end{array}$ & ND & $21,6 \pm 7,1$ & ND & $17,4 \pm 6,2$ & $20,4 \pm 7,0$ \\
\hline $\begin{array}{l}\text { Markland e } 0 \text { liverer } \\
\text { Inglaterra }\end{array}$ & $\begin{array}{l}369 \text { 우 } \\
\text { (18-35 anos) }\end{array}$ & ND & $\begin{array}{l}25,8 \pm 7,8 \\
\alpha=, 90\end{array}$ & $\begin{array}{l}14,3 \pm 4,6 \\
\alpha=, 84\end{array}$ & $\begin{array}{l}19,7 \pm 6,4 \\
\alpha=, 85\end{array}$ & $\begin{array}{l}25,7 \pm 6,8 \\
\alpha=, 89\end{array}$ \\
\hline Swami ${ }^{32}$ - Malásia & $\begin{array}{l}554+ \\
\text { (18-76 anos) }\end{array}$ & ND & $\alpha=, 94$ & $\alpha=, 82$ & $\alpha=, 94$ & $\alpha=, 88$ \\
\hline Swami ${ }^{29}$ - Inglaterra & $\begin{array}{l}322 \text { + universitárias } \\
\text { (média de } 24,7 \text { anos) }\end{array}$ & ND & $\begin{array}{l}27,9 \pm 4,6 \\
\alpha=, 93\end{array}$ & $\begin{array}{l}13,8 \pm 3,9 \\
\alpha=, 91\end{array}$ & $\begin{array}{l}23,5 \pm 5,4 \\
\alpha=, 83\end{array}$ & $\begin{array}{l}27,7 \pm 4,7 \\
\alpha=, 87\end{array}$ \\
\hline Dunker ${ }^{11}$ - Brasil & $\begin{array}{l}48 \text { } ~ \\
\text { (12-14 anos) } \\
\text { (fase pré-intervenção) }\end{array}$ & $95,5 \pm 12,9$ & $27,7 \pm 5,2$ & $15,5 \pm 2,2$ & $22,8 \pm 4,4$ & $29 \pm 3,9$ \\
\hline Dunker et al. ${ }^{22}$ - Brasil & $\begin{array}{l}183 \text { क } \\
\text { (15-18 anos) }\end{array}$ & $88 \pm 15,5$ & ND & ND & ND & ND \\
\hline
\end{tabular}

ND: dado não disponível no estudo. 
Jordânia escores menores que os do presente estudo em todas subescalas; e Wilksch et al..$^{30}$ observaram que o escore total e SUB4 na fase pré-intervenção com adolescentes foram menores do que os do presente estudo.

Cusumano e Thompson ${ }^{27}$ avaliaram universitárias por meio da versão mais antiga da SATAQ em relação à imagem corporal e ao corpo ideal mostrados em revistas e concluíram que a exposição a essas imagens não teve valor preditivo, mas que a consciência das pressões culturais teve correlação com a imagem corporal e que a internalização das normas sociais de aparência foi a responsável pela variância significativa e substancial na imagem corporal.

Em estudos realizados com mulheres inglesas (universitárias ou majoritariamente universitárias), os valores de todas as subescalas da SATAQ foram maiores que os do presente estudo 28,29

Nacionalmente, os estudos que utilizaram a SATAQ realizaram avaliações apenas com adolescentes. Dunker ${ }^{11}$, avaliando meninas, encontrou na fase de pré-intervenção valores maiores do que os do presente estudo (com exceção da Informação); e numa avaliação de comportamentos de risco para TA em meninas encontrou-se uma média de escore total na SATAQ próxima do valor do presente estudo ${ }^{22}$.

A identificação de fatores de risco para insatisfação corporal e TA é essencial para o desenvolvimento e a implantação de programas efetivos de tratamento e prevenção ${ }^{13}$. Estudo nessa área demonstrou que a internalização da mídia pode ser um fator de risco causal para o início de problemas com a alimentação e o peso ${ }^{10}$; a pressão da mídia influenciaria fortemente as tendências do indivíduo para adotar atitudes e comportamentos alimentares desordenados ${ }^{26}$. O'Riordan e Zamboanga ${ }^{26}$ encontraram que a pressão da mídia mediou a associação entre a internalização e as tendências para bulimia nervosa entre universitários e que a pressão pareceu ter um papel maior do que a informação ou uso da mídia como fonte de informação.

Harrison e Cantor ${ }^{2}$ encontraram que o uso da mídia foi um preditor de sintomatologia de TA, busca pela magreza e insatisfação corporal em universitários americanos. Segundo Thompson et al. ${ }^{13}$, pode ser útil conceituar a mídia como uma grande influência de massa, talvez tendo um papel na formação e na adoção de padrões idealizados de aparência, pelos pais, amigos e outros agentes de influência (professores, treinadores).

Uma abordagem promissora em programas de prevenção dos TA voltada ao fator sociocultural é chamada de "Media Literacy", ou educação sobre a mídia. São programas baseados na teoria cognitivo-comportamental que buscam reduzir os fatores de risco para os TA por meio de atividades interativas que ajudam os participantes a adotarem uma visão crítica sobre a mídia, propondo alternativas aos ideais culturais apresentados por ela ${ }^{30}$.

Algumas limitações devem ser discutidas sobre este estudo; não foi usado para cálculo de amostragem o desfecho "influência da mídia", e sim uma estimativa de atitudes alimentares desordenadas. De qualquer forma, este tema não é tradicionalmente estudado no Brasil e não há estimativa nacional sobre influência da mídia. Mas, dado o número significativo de participantes e o fato de que não existem estudos similares no país, um perfil da influência da mídia para mulheres jovens e suas diferenças regionais pode ser obtido e deve ser explorado em pesquisas futuras. A amostra do estudo não foi probabilística, mas considera-se que a resposta de 37 instituições de ensino para parceria nesta pesquisa foi expressiva, considerando-se as problemáticas com a logística de envio e reenvio dos questionários e a aplicação deles ao público-alvo. Em relação à participação assimétrica entre as regiões, deve-se considerar que a distribuição das instituições de ensino no país é também assimétrica, com um número menor no Norte e no Centro-Oeste ${ }^{31}$.

Outra questão a se considerar é o instrumento utilizado, apenas traduzido e não validado para o português e cenário nacional. No entanto, a consistência interna obtida no instrumento em geral e a obtida nas subescalas nesta avaliação $(1=0,86 ; 2=0,68 ; 3=0,87 ; 4=0,77)$ foram adequadas $\mathrm{e}$ similares ao estudo original de desenvolvimento da SATAQ ${ }^{13}$ (Quadro 1) - que foi utilizado em muitos países, em estudos de imagem corporal e TA, apresentando boa consistência interna em amostras clínicas e não clínicas com uma adequada validade convergente ${ }^{4}$.

Segundo Cafri et al. ${ }^{4}$, as subescalas da SATAQ demonstram ter uma forte relação com a imagem corporal ou práticas de controle de peso. No entanto, alguns estudos em culturas não ocidentais tiveram resultados controversos: na Jordânia, a escala apresentou-se estável, indicando ser um bom instrumento para avaliar a influência da mídia na imagem corporal ${ }^{17}$, mas estudo com mulheres malasianas e chinesas indicou inadequação do instrumento, sugerindo a elaboração de uma escala loca| ${ }^{32}$. Acredita-se que a cultura brasileira é mais similar ao padrão americano do que ao padrão oriental. De qualquer forma, sugere-se que estudos de avaliação psicométrica e análise fatorial confirmatória sejam realizados com a versão em português da SATAQ.

A influência da mídia deve ser considerada em programas de educação nutricional, abordagem da insatisfação corporal e de prevenção para TA. Educadores e profissionais da área da saúde devem ampliar seus conhecimentos sobre a influência da mídia e seu papel nos TA e na imagem corporal, para que possam incorporar esses temas em seus programas de prevenção e tratamento.

\section{CONCLUSÃO}

Universitárias brasileiras têm influência da mídia em grau similar, mesmo vivendo em diferentes regiões do país. Estudantes do Sul apresentaram maior aceitação das mensagens 
da mídia em relação a ideais estéticos não realistas, e as do Nordeste consideraram a mídia uma fonte de informação importante sobre a aparência mais do que as demais. Estudantes mais jovens, com excesso de peso e com maior renda foram em média mais influenciadas pela mídia.

\section{AGRADECIMENTOS}

Os autores agradecem à Fundação de Amparo à Pesquisa do Estado de São Paulo - FAPESP (processo 06/56850-9) pelo apoio à realização desta pesquisa.

Os autores agradecem também pela expressiva participação das instituições: Centro de Ensino Unificado de Teresina, Centro Universitário de Barra Mansa, Centro Universitário de Brasília, Centro Universitário de Vila Velha, Centro Universitário de Volta Redonda, Centro Universitário do Sul de Minas, Centro Universitário Feevale, Centro Universitário La Salle, Centro Universitário Nilton Lins, Faculdade Itabirana de Saúde, Faculdade Natalense para o Desenvolvimento do Rio Grande do Norte, Faculdade São Lucas, Faculdade Vale do Ipojuca, Pontifícia Universidade Católica do Paraná, Unidade de Ensino Superior do Sul do Maranhão, Universidade Católica Dom Bosco, Universidade Cruzeiro do Sul, Universidade de Brasília, Universidade de Caxias do Sul, Universidade de Cuiabá, Universidade do Sul de Santa Catarina, Universidade do Vale do Itajaí, Universidade do Vale do Rio dos Sinos, Universidade Federal de Alfenas, Universidade Federal de Pelotas, Universidade Federal de Santa Catarina, Universidade Federal do Mato Grosso, Universidade Federal do Paraná, Universidade Federal do Piauí, Universidade Federal do Rio Grande do Sul, Universidade Metodista de Piracicaba, Universidade Metodista de São Paulo, Universidade Municipal de São Caetano do Sul, Universidade Paulista Campus Manaus/São Paulo - Capital/Sorocaba.

\section{REFERÊNCIAS}

1. McLuhan M, Quentin F. The medium is the massage: an inventory of effects. New York: Bantam Books; 1967.

2. Harrison $\mathrm{K}$, Cantor J. The relationship between media consumption and eating disorders. J Commun. 1997;47:40-67.

3. Ditamar H. Vulnerability factors and processes linking sociocultural pressures and body dissatisfaction. J Soc Clin Psychol. 2005;24:1081-7.

4. Cafri G, Yamamiya Y, Brannick M, Thompson JK. The influence of sociocultural factors on body image: a meta-analysis. Clinical Psychology: Science and Practice. 2005;12:421-33.

5. Stice E, Schupak-Neuberg E, Shaw HE, Stein RI. Relation of media exposure to eating disorder symptomatology: an examination of mediating mechanisms. J Abnorm Psychol. 1994;103:836-40.

6. American Psychiatry Association. Practice Guideline for the treatment of patients with eating disorders. 3. ed. Virginia: American Psychiatric Publishing; 2006.

7. Dunker KLL. Prevenção dos transtornos alimentares: uma revisão metodológica. Nutrire: Rev Soc Bras Alim Nutr. 2009;34:195-211.
8. Irving LM, Neumark-Sztainer D. Integrating the prevention of eating disorders and obesity: feasible or futile? Prev Med. 2002;34:299-309.

9. Alvarenga MS, Scagliusi FB, Philippi ST. Comparação das atitudes alimentares entre universitárias das cinco regiões brasileiras. (ien Saude Colet. 2010. [aceito]

10. Thompson JK, Stice E. Thin-Ideal internalization: mounting evidence for a new risk factor for body-image disturbance and eating pathology. Curr Dir in Psychol Sci. 2001;10:181-3.

11. Dunker KLL. Programa de prevenção de comportamentos de risco para transtornos alimentares em adolescentes: estudo piloto [tese]. São Paulo: FCF/FEA/FSP/USP; 2006.

12. World Health Organization (WHO). Global database on Body Mass Index. Geneva: WH0; 2006. (accessed 15 March 2009). Available from: http://apps.who.int/bmi/index. jsp?introPage=intro_3.html.

13. Thompson JK, Berg PVD, Roehrig M, Guarda AS, Heinber LJ. The sociocultural attitudes towards apperance scale-3 (SATAQ-3): development and validation. Int J Eat Disord. 2004;35:293-304.

14. Alvarenga MS, Lourenço BH, Sato PM, Scagliusi FB, Philippi ST. Insatisfação com a imagem corporal em universitárias brasileiras. J Bras Psiquiatr. 2010;59(1):44-51.

15. Alvarenga MS, Scagliusi FB, Philippi ST. Comportamento de risco para transtorno alimentar em universitárias brasileiras. Rev Psiq Clin. 2010, no prelo.

16. Groez LM, Levine MP, Murner SK. The effect of experimental presentation of thin media images on body satisfaction: a meta-analytic review. Int J Eat Disord. 2002;31:1-16.

17. MadanatHN, Hawks SR, Brown RB. Validation of the Sociocultural Attitudes Towards Appearance Questionnaire-3 among a random sample of Jordanian women. Body Image. 2006;3:421-5.

18. Neighbors LA, Sobal J. Prevalence and magnitude of body weight and shape dissatisfaction among university students. Eat Behav. 2007;8:429-39.

19. Martinez-Gonzalez MA, Gual P, Lahortiga F, Alonso Y, Irala-Estévez J, Cervera S. Parental factors, mass media influences, and the onset of eating disorders in a prospective population-based cohort. Pediatrics. 2003;111:315-20.

20. Serra GMA, Santos LM. Saúde e mídia na construção da obesidade e corpo perfeito. Cien Saude Colet. 2003;8:691-701

21. Rodin J, Silberstein L, Striegel-Moore RH. Women and weight: a normative discontent. In: Sonderegger TB, Anastasi A, ed. Psychology and gender. Lincoln, NE: University of Nebraska Press; 1985, p. 245-307.

22. Dunker KLL, Fernandes CPB, Carreira Filho D. Influência do nível socioeconômico sobre comportamentos de risco para transtornos alimentares em adolescentes. J Bras Psiquiatr. 2009;58:156-61.

23. Snapp $\mathrm{S}$. Internalization of the thin ideal among low-income ethic minority adolescent girls. Body Image. 2009;6:311-4.

24. Monro F, Huon G. Media-portrayed idealized images, body shame, and appearance anxiety. Int J Eat Disord. 2005;38:85-90.

25. Shroff $H$, Thompson JK. Body image and eating disturbance in India: media and interpersonal influences. Int J Eat Disord. 2004;35:198-203.

26. O'Riordan SS, Zamboanga BL. Aspects of the media and their relevance to bulimic attitudes and tendencies among female college students. Eat Behav. 2008;9:247-50.

27. Cusumano DL, Thompson JK. Body Image and body shape ideals in magazines: exposure, awareness, and internalization. Sex Roles. 1997;37:701-21.

28. Markland D, Oliver EJ. The sociocultural attitudes towards appearance questionnaire-3: a confirmatory factor analysis. Body Image. 2008;5:116-21.

29. Swami V. Body appreciation, media influence, and weight status predict consideration of cosmetic surgery among female undergraduates. Body Image. 2009;6:315-7.

30. Wilksch SM, Tiggemann M, Wade TD. Impact of interactive school-based media literacy lessons for reducing internalization of media ideals in young adolescent girls and boys. Int J Eat Disord. 2006;39:385-93.

31. Ministério da Educação. Senso da Educação Superior. Instituto Nacional de Estudos e Pesquisas Educacionais Anísio Teixeira. Sinopses Estatísticas da Educação Superior - Graduação, 2007. Disponível em: http://www.inep.gov.br/superior/censosuperior/sinopse/default.asp.

32. Swami V. An examination of the factor structure of the Sociocultural Attitudes Towards Appearance Questionnaire-3 in Malaysia. Body Image. 2009;6:129-32. 